\title{
Hausstaubmilben: Allergenkarenz lindert Allergiesymptome
}

\section{Maßnahmen zur Reduktion von Allergenen der Hausstaubmilbe haben positive Effekte auf die Allergiesymptomatik, vor allem des Asthma bronchiale. Sie bremsen auch in gewissem Maße die Ausbildung einer Sensibilisierung, können aber der heutigen Studienlage zufolge nicht nachweisbar den Ausbruch eines Asthma im Sinne einer Primärprävention verhindern.}

Hausstaubmilben mit ihren Allergenen gelten in den industrialisierten Ländern als eine der Hauptursachen für die Entwicklung einer perennialen Rhinitis oder eines Asthma bronchiale. Der Reduktion dieser Innenraumallergene kommt deshalb ein hoher Stellenwert zu. Über die Wirksamkeit von Eliminationsmaßnahmen auf den unterschiedlichen Stufen der Erkrankung besteht mittlerweile Konsens.

\section{Wert der Tertiärprävention ist gesichert}

Querschnitts- und Longitudinalstudien zeigen, so S. Lau (Berlin), daß Eliminationsmaßnahmen von Hausstaubmilbenallergenen aus dem häuslichen Milieu in Form einer Sekundär- und Tertiärprävention wirksam sind. Lau definierte dabei die drei Stufen der Prävention wie folgt: Die Primärprävention ist ausgerichtet auf die Vermeidung der Entwicklung einer Sensibilisierung, bei der Sekundärprävention wird die Ausbildung von Allergiesymptomen nach stattgefundener Sensibilisierung angestrebt und in der Tertiärprävention steht die Symptomkontrolle bei der klinisch manifest gewordenen Allergie im Vordergrund.

Die Zusammenfassung von Untersuchungen zur Tertiärprävention bei Kindern mit einem manifesten Asthma bronchiale attestiert den verschiedenen heute praktizierten Präventionsmaßnahmen eine unterschiedlich hohe
Wirksamkeit. Nach Laus Worten ist die therapeutische Effektivität von „Encasings", also milbenallergendichten Matratzenbezügen, unbestritten und auch in plazebokontrollierten Studien anhand des objektiven Parameters bronchiale Hyperreaktivität gut belegt. Der zusätzliche Einsatz von Luftfiltern kann einer anderen Untersuchung zufolge empfohlen werden.

\section{Bewährte Präventionsmaßnahmen}

Akarizide weisen in In-vivo-Versuchen eine hohe milbentötende Wirksamkeit, die diese Stoffe aber unter Praxisbedingungen höchstens über 2 Tage aufbringen. Staubsaugen, wie es heute in Haushalten üblich ist, bringt ebenfalls nicht viel, so Lau. Eine Entfernung von Teppichböden gehört dagegen zum Empfehlungsrepertoire von Pädiatern ebenso wie der Rat, Kuscheltiere über 2 Tage einzufrieren, was für einige Zeit die Milbenkonzentration reduziert. Verzichtet werden sollte gerade in den Wintermonaten auf den Einsatz eines bung der relativen Luftfeuchtigkeit zusammen mit der Heizungswärme für ein optimales Gedeihen der Milben sorgt. Auch an regelmäßiges Lüften sollte gedacht werden.

Für die Primärprävention, also der Vermeidung einer Sensibilisierung geStudienlage zu diesen Milbeneliminationsmaßnahmen nicht so eindeutig. Luftbefeuchters, der über die Anhegen Hausstaubmilbenallergene, ist die

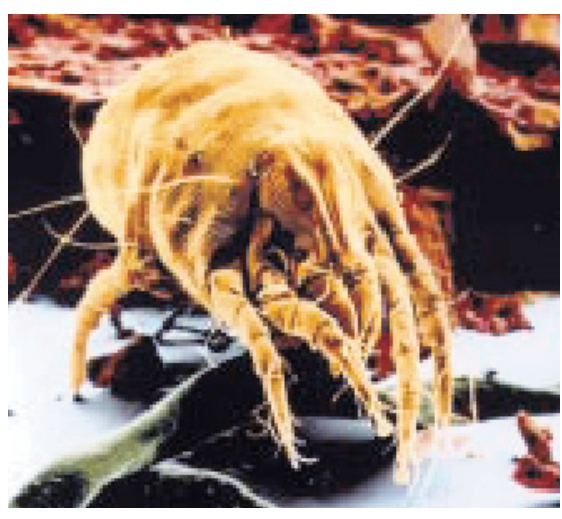

Die Ausscheidungen der Hausstaubmilbe stehen im Mittelpunkt der epidemiologischen Forschungen zur Allergieentwicklung. Eine Allergenreduktion sollte in jedem Fall angestrebt werden.

Lau: „Da kommen wir auf ein unsicheres Terrain." Zwar ergibt sich bei der Reduktion von Inhalationsallergenen bei Kindern im ersten Lebensjahr statistisch ein Trend $\mathrm{zu}$ einer geringeren Sensibilisierung, bei den respiratorischen Symptomen ist aber kaum mehr ein Effekt nachweisbar.

\section{Multifaktorieller \\ Krankheitsausbruch}

So kann ein gewisser präventiver Effekt auf die Ausbildung einer Sensibilisierung gerade bei familiär belasteten Risikokindern nachgewiesen werden. „Wir wissen aber nicht, ob sich damit wirklich der Ausbruch einer Asthmasymptomatik vermeiden läßt", so Lau. „Hier spielen noch viele andere Faktoren eine Rolle, deren Bedeutung wir gerade erst in Ansätzen erfassen." Neben einer genetischen und psychischen Veranlagung kommen auch exogene Einflüsse in Frage. So ist z.B. momentan die Bedeutung von Infektionen im ersten Lebensjahr Gegenstand intensiver Forschungen.

Besorgten Eltern von Risikokindern kann daher zu Milbensanierungsmaßnahmen geraten werden, in der Hoffnung, daß die Kinder weniger gegen die Allergene sensibilisiert werden. Eine Garantie, daß diese Kinder dadurch in ihrem späteren Leben vor Asthma geschützt sind, sollte dagegen niemand geben.

S. Lau (Berlin) anläßlich der 21. Tagung der Deutschen Gesellschaft für Allergologie und klinische Immunologie, München 1999. 